\title{
Simulation of Multiphysics Multiscale Systems, 5th International Workshop
}

\author{
Valeria V. Krzhizhanovskaya ${ }^{1,2}$ and Alfons G. Hoekstra ${ }^{1}$ \\ ${ }^{1}$ Section Computational Science, Faculty of Science, University of Amsterdam, \\ Kruislaan 403, 1098 SJ Amsterdam, The Netherlands \\ http://www.science.uva.nl/ valeria/SMMS \\ \{valeria, alfons\} ascience.uva.nl \\ ${ }^{2}$ St. Petersburg State Polytechnic University, Russia
}

\begin{abstract}
Modeling and Simulation of Multiphysics Multiscale Systems (SMMS) poses a grand challenge to computational science. To adequately simulate numerous intertwined processes characterized by different spatial and temporal scales spanning many orders of magnitude, sophisticated models and advanced computational techniques are required. The aim of the SMMS workshop is to encourage and review the progress in this multidisciplinary research field. This short paper describes the scope of the workshop and gives pointers to the papers reflecting the latest developments in the field.
\end{abstract}

Keywords: Multiphysics, Multiscale, Complex systems, Modeling, Simulation, ICCS, SMMS, Workshop.

\section{Introduction to the Workshop}

The progress in understanding physical, chemical, biological, sociological and economical processes strongly depends on adequacy and accuracy of numerical simulation. All the systems important for scientific and industrial applications are inherently multiphysics and multiscale: they involve interactions amongst a wide range of physical phenomena operating at different spatial and temporal scales. Complex flows, fluid-structure interactions, plasma and chemical processes, thermo-mechanical and electromagnetic systems are just a few examples essential for fundamental and applied sciences. Numerical simulation of these multiphysics and multiscale problems requires development of sophisticated models and methods for their integration, as well as efficient numerical algorithms and advanced computational techniques.

To boost scientific cross-fertilization and promote collaboration of the diverse groups of specialists involved, we have launched a series of mini-symposia on Simulation of Multiphysics Multiscale Systems (SMMS) in conjunction with the International Conference on Computational Sciences (ICCS) [1].

The fifth workshop in this series, organized as a part of ICCS-2008, expands the scope of the meeting from physics and engineering to biological and biomedical applications. This includes computational models of tissue- and organo-genesis, tumor growth, blood vessel formation and interaction with the hosting tissue, biochemical 
transport and signaling, biomedical simulations for surgical planning, etc. The topics traditionally addressed by the symposium include modeling of multiphysics and/or multiscale systems on different levels of description, novel approaches to combine different models and scales in one problem solution, advanced numerical methods for solving multiphysics multiscale problems, new algorithms for parallel distributed computing specific to the field, and challenging multiphysics multiscale applications from industry and academia.

A large collection of rigorously reviewed papers selected for the workshops highlight modern trends and recent achievements [2]. It shows the progress made in coupling different models (such as continuous and discrete models; quantum and classical approaches; deterministic and stochastic techniques; nano, micro, meso and macro descriptions) and suggests various coupling approaches (e.g. homogenization techniques, multigrid and nested grids methods, variational multiscale methods; embedded, concurrent, integrated or hand-shaking multiscale methods, domain bridging methods, etc.). A number of selected papers have been published in the special issues of the International Journal for Multiscale Computational Engineering [3], collecting state-of-the-art methods for multiscale multiphysics applications.

Acknowledgments. We would like to thank the participants of our workshop for their inspiring contributions, and the members of the workshop program committee for their diligent work, which led to the very high quality of the conference. The organization of this event was partly supported by the Virtual Laboratory for e-Science Bsik project.

\section{References}

1. Simulation of Multiphysics Multiscale Systems, http: / /www.science.uva.nl/ valeria/SMMS

2. LNCS this volume, 20 papers after this introduction;

LNCS V. 4487/2007. DOI 10.1007/978-3-540-72584-8, pp. 755-954;

LNCS V. 3992/2006. DOI 10.1007/11758525, pp. 1-138;

LNCS V. 3516/2005. DOI 10.1007/b136575, pp. 1-146;

LNCS V. 3039/2004. DOI 10.1007/b98005, pp. 540-678

3. Simulation of Multiphysics Multiscale Systems. Special Issues of the International Journal for Multiscale Computational Engineering:

V. 4, Issue 2, 2006. DOI: 10.1615/IntJMultCompEng.v4.i2;

V. 4, Issue 3, 2006. DOI: 10.1615/IntJMultCompEng.v4.i3;

V. 5, Issue 1, 2007. DOI: 10.1615/IntJMultCompEng.v5.i1;

V. 6, Issue 1, 2008. DOI: 10.1615/IntJMultCompEng.v6.i1 